\title{
INTERVENÇÃO DE ENFERMAGEM NO ESTRESSE DO CUIDADOR FAMILIAR DO IDOSO COM DEPENDÊNCIA: ESTUDO PILOTO
}

\author{
NURSING INTERVENTION FOR STRESS MANAGEMENT \\ IN FAMILY CAREGIVERS OF DEPENDENT OLDER \\ ADULTS: A PILOT STUDY
}

\section{INTERVENCIÓN DE ENFERMERÍA EN EL ESTRÉS DEL CUIDADOR FAMILIAR DEL ANCIANO CON DEPENDENCIA: ESTUDIO PILOTO}

\author{
Laura Maria Viegas ${ }^{1}$ \\ Ana Alexandre Fernandes ${ }^{2}$ \\ Maria dos Anjos Pereira Lopes F. Veiga ${ }^{3}$
}

Como citar este artigo: Viegas LM, Fernandes AA, Veiga MAPL. Intervenção de enfermagem no estresse do cuidador familiar do idoso com dependência: estudo piloto. Rev baiana enferm. 2018;32:e25244.

Objetivo: avaliar a eficácia de uma intervenção de enfermagem estruturada com base num programa psicoeducativo de gestão do estresse sobre estressores primários (sobrecarga), recursos (coping) e resultados (bem-estar emocional) do cuidador, e avaliação realizada pelos cuidadores e pelos enfermeiros sobre a ajuda da intervenção e sobre o material de apoio ao programa. Método: estudo piloto com 13 cuidadores de idosos em Portugal durante 5 semanas. Realizaram-se entrevistas para avaliar a intervenção e o material de apoio. Resultados: depois da intervenção, houve melhoria no coping, no bem-estar e na sobrecarga com diferença estatisticamente significativa na sobrecarga; dificuldades com a implementação da intervenção e uso do material de apoio. Conclusão: os cuidadores familiares revelaram que a intervenção ajudou-os na aprendizagem de novas estratégias de coping. Por parte dos enfermeiros, a intervenção ajudou-os a conhecer as dificuldades do cuidador, facilitando a abordagem holística do cuidado centrado no cuidador e no idoso.

Descritores: Intervenção de enfermagem no domicílio. Estresse. Cuidador familiar. Idoso.

Objective: assess the effectiveness of a structured nursing intervention for caregivers based on a psychoeducational stress management program in relation to primary stressors (overload), resources (coping) and results (emotional well-being), as well as an assessment by caregivers and care recipients as to the helpfulness of the intervention and the program's support material. Method: a pilot study was conducted with 13 caregivers of older adults in Portugal during five weeks. Interviews were conducted to assess the intervention and support material. Results: after the intervention, there was an improvement in coping, well-being and overload, with a statistically significant difference in overload; difficulties implementing the intervention and using the support material. Conclusion: the family caregivers stated that the intervention helped them learn new coping strategies. On the part of the nurses, the

\footnotetext{
Enfermeira. Mestre em Psicologia Clínica. Doutoranda do Curso de Doutoramento em Enfermagem na Universidade de Lisboa. Professor adjunto no Departamento de Fundamentos de Enfermagem na Escola Superior de Enfermagem de Lisboa. Lisboa, Portugal. Iviegas@esel.pt

Doutora em Sociologia. Professora Catedrática no Instituto Superior de Ciências Sociais e Políticas da Universidade Técnica de Lisboa. Lisboa, Portugal.

Doutora em Ciências da Enfermagem. Professora da Escola Superior de Enfermagem de Lisboa (aposentada). Investigadora na Unidade de Investigação e Desenvolvimento em Enfermagem. Lisboa, Portugal.
} 
intervention belped them understand the difficulties faced by caregivers, facilitating a bolistic care approach based on the caregiver and elderly person.

Descriptors: Household nursing intervention. Stress; Family caregiver Older adult.

Objetivo: evaluar la eficacia de una intervención de enfermería estructurada con base en un programa psicoeducativo de gestión del estrés sobre estresores primarios (sobrecarga), recursos (coping) y resultados (bienestar emocional) del cuidador, y evaluación realizada por cuidadores y enfermeros sobre la ayuda de la intervención y el material de apoyo al programa. Método: estudio piloto con 13 cuidadores de ancianos en Portugal, durante cinco semanas. Se realizaron entrevistas para evaluar intervención y material de apoyo. Resultados: después de la intervención, hubo mejora en el coping, bienestar y sobrecarga, con diferencia estadisticamente significativa en la sobrecarga; dificultades con la implementación de la intervención y uso del material de apoyo. Conclusión: los cuidadores familiares revelaron que la intervención ayudó en el aprendizaje de nuevas estrategias de coping. Para los enfermeros, ayudó a conocer las dificultades del cuidador, facilitando el abordaje bolístico de la atención centrada en el cuidador y anciano.

Descriptores: Intervención de enfermería en el domicilio. Estrés. Cuidador. Anciano.

\section{Introdução}

Portugal é um dos países mais envelhecidos do mundo e também da Europa. Na última década, houve um recuo acentuado da natalidade $^{(1)}$. No último recenseamento ${ }^{(2)}$, a população portuguesa com mais de 65 anos já era 19,1\% do total, dos quais 21,5\% eram mulheres e 16,8\% eram homens. A esperança de vida à nascença é também uma das mais elevadas da Europa. As mulheres portuguesas nascidas em 2016 esperam poder viver até os 84 anos e os homens até os 78 anos $^{(3)}$.

O crescimento da longevidade humana acarreta o aumento das necessidades de saúde ao longo do processo de envelhecimento, com a diminuição da funcionalidade e os seus impactos nas actividades de vida diária. No âmbito da vida familiar, são os cônjuges ou outros parentes próximos que prestam os cuidados. A dinâmica da prestação de cuidados tem uma dimensão temporal à qual o cuidador familiar vai adaptando-se, à medida que a funcionalidade reduz e as necessidades aumentam. A Family Caregiver Alliance $^{(4)}$ descreve o caregiving como um processo dinâmico experienciado pelo cuidador familiar durante o desempenho do seu papel num continuum de cuidados. Esse processo, designado caregiving, leva o cuidador à exposição de uma elevada carga física e psicológica perante a qual pode incorrer num conjunto de sintomas que constituem um padrão disfuncional identificado como "estresse do prestador de cuidados" ou "sobrecarga do papel do cuidador" (5).

O cuidador é normalmente um familiar, amigo ou vizinho que, no quotidiano, presta cuidados a uma pessoa receptora de cuidados com doença crónica ou incapacitante que a impede de realizar as actividades de vida diária, total ou parcialmente, tornando-a, por vezes, dependente. É um recurso não institucional para o cuidar $^{(6)}$, por não ser remunerado habitualmente pelo cuidado prestado ${ }^{(7)}$.

As exigências da prestação de cuidados levam a olhar para o cuidador, tendo em vista a necessidade de preservar o seu papel social e prevenir o risco de adoecer. Para tal é necessário desenvolver intervenções que o ajudem a manter a sua saúde ${ }^{(8)}$, considerando que as pessoas preferem manter-se em casa, perto da família ${ }^{(9)}$. Esses cuidados contribuem também para diminuir o risco de institucionalização e reduzir os custos dos cuidados de longa duração ${ }^{(6)}$. A literatura evidencia que as intervenções psicoeducativas são promotoras do bem-estar do cuidador familiar ${ }^{(10)}$. Há também evidência da necessidade de os profissionais de saúde apoiarem o cuidador familiar no desempenho do seu papel.

Entretanto, a intervenção de enfermagem é mais eficaz quando é individualizada e efectuada por meio da recolha de informação sobre as características da pessoa, suas necessidades 
e percepções, da qual decorrem acções em que a pessoa cuidada participa na tomada de decisão sobre qual deve ser a forma mais ajustada a $\mathrm{si}^{(11)}$.

$\mathrm{Na}$ avaliação do estresse do cuidador, autor ${ }^{(12)}$ defende o uso do modelo transaccional de estresse de Pearlin, Mullan, Semple e Skaff. Esta opção baseia-se no pressuposto de que as várias dimensões desse modelo operacionalizam a variabilidade na forma como o cuidador vivencia a sua experiência de cuidar. São as seguintes as dimensões do modelo: contexto de cuidado (características sociodemográficas do cuidador e da pessoa cuidada e história da prestação de cuidados); estressores primários e secundários; recursos e resultados. A elaboração do modelo baseou-se na concepção teórica ${ }^{(13)}$ que considera o estresse como resultado de uma transacção entre o indivíduo e o ambiente, que excede os recursos do indivíduo, necessitando de esforço para se adaptar. De acordo com essa concepção, coping refere-se aos esforços cognitivos e comportamentais em constante mudança na gestão de questões externas/internas específicas, as quais são avaliadas por excederem os recursos já referidos. Por isso, é importante o indivíduo manter a capacidade para aprender e usar novas estratégias, de acordo com novas necessidades, desenvolvendo diferentes estilos de coping focados na resolução de problemas ou nas emoções. O enfermeiro pode contribuir, junto do cuidador, no reconhecimento de estratégias para alívio da sobrecarga e no desenvolvimento de estratégias para a diminuição do estresse ${ }^{(14)}$.

Em Portugal, os estudos mostram que as intervenções do enfermeiro junto do cuidador visam, sobretudo, a melhoria das suas competências na prestação de cuidados, tornando-o o "parceiro oculto". Nessa dinâmica, é frequente que sejam subvalorizadas as suas necessidades decorrentes do papel de cuidador, sendo reiterada somente a referência a comentários, como "lembre-se de cuidar de si”. Assim, as intervenções estruturadas com base em programas psicoeducativos dirigidas ao estresse do cuidador, além de limitadas e pouco frequentes, devem ser avaliadas quanto à sua eficácia. Os resultados de tal avaliação contribuem para melhorar os cuidados de enfermagem e as competências e eficiência do acto de cuidar do cuidador familiar com efeitos positivos na sua saúde.

A hipótese considerada neste estudo é que a intervenção estruturada num programa reduza a sobrecarga, aumente o coping e o bem-estar emocional. Espera-se a adequação do material de apoio ao programa.

O objectivo central deste estudo piloto consiste em avaliar a eficácia de uma intervenção de enfermagem estruturada com base num programa psicoeducativo de gestão do estresse sobre estressores primários (sobrecarga), recursos (coping) e resultados (bem-estar emocional) do cuidador, bem como a avaliação realizada pelos cuidadores e pelos enfermeiros sobre a ajuda da intervenção e sobre o material de apoio ao programa.

\section{Método}

Trata-se de um estudo piloto observacional longitudinal prospectivo. A amostra de conveniência foi constituída por cuidadores de familiares idosos em situação de dependência funcional que se encontravam inscritos em apoio domiciliário nas Unidades de Saúde de um Agrupamento de Centros de Saúde da Sub Região de Saúde de Lisboa e Vale do Tejo, Portugal.

A observação ocorreu entre dezembro de 2014 e março de 2015 e teve como critérios de inclusão: cuidadores familiares que assumiam a responsabilidade pelos cuidados com score $\leq 16$ na Grelha para avaliação do cuidador em risco ${ }^{(15)}$, idade superior a 18 anos, saber ler e escrever e com contacto telefónico.

$\mathrm{Na}$ visita de enfermagem domiciliária, os enfermeiros contactaram os cuidadores familiares elegíveis e explicaram os objetivos do estudo. $\mathrm{Na}$ visita seguinte, foi explicado o protocolo de investigação, pedido o consentimento informado, aplicada a "Grelha para avaliação do cuidador em risco" e combinado o momento para iniciar a intervenção estruturada de enfermagem.

A intervenção de enfermagem no domicílio, para o cuidador familiar com dependência, foi suportada pelo programa psicoeducativo do 
processo de gestão do estresse de Ducharme, Trudeau e Ward ${ }^{(15)}$ : Gestion du stress-programme psychoéducatif de gestion du estress destiné aoux proches-aidants d'un parent âgé à domicile. A opção por um programa de gestão de estresse, já publicado internacionalmente, deveu-se a estar alicerçado no conceito de estresse e coping ${ }^{(13)}$. Além disso, essa opção ampara-se no facto de o programa ser composto por etapas que, ao corresponderem ao processo de gestão do estresse, permite ao cuidador a tomada de decisão sobre a escolha de uma situação estressante para si na prestação de cuidador, privilegiando, desse modo a individualização, assim como a aprendizagem, para gerir outras situações estressantes a surgir durante o percurso do seu papel de cuidador.

Feito contato via e-mail com uma das autoras, que autorizou a utilização do referido programa e como adquirir o material de apoio: caderno do cuidador e manual de aplicação. Foram feitos os procedimentos na adaptação para a língua portuguesa do material escrito: equivalência conceptual e linguística.

O referido programa psicoeducativo tem como objetivo promover competências dos cuidadores para contribuir para uma melhor gestão de situações difíceis/estressantes vividas na prestação de cuidados ao familiar no domicílio.

O processo de gestão do estresse do programa desenvolve-se em cinco etapas:

$1^{\underline{a}}$ etapa - tomada de consciência. O cuidador descreve a situação geral de prestação de cuidados: refere aspetos positivos e aspetos difíceis vividos na situação de prestação de cuidados;

$2^{\text {a }}$ etapa - escolha de uma situação difícil/ estressante e de um objetivo a atingir. O cuidador escolhe uma situação difícil/estressante experienciada na prestação de cuidados que pretende mudar. $\mathrm{O}$ cuidador formula um objetivo a atingir com vistas a reduzir a sua perceção face a essa situação que escolheu, com a ajuda da "Escala para atingir os objetivos pessoais". Este instrumento permitirá avaliar, no final da 5- etapa, se o objetivo identificado foi ou não atingido; $3^{\text {a }}$ etapa - análise da situação escolhida. O cuidador reflete sobre a situação difícil/estressante escolhida e os fatores que influenciam a forma de a perceber (pessoais, familiares e contextuais) e quais as estratégias habitualmente usadas;

4- etapa - escolha de uma estratégia ajustada à situação escolhida e praticá-la. O cuidador é convidado a experimentá-la(s) de entre a(s) estratégia(s) adaptativa(s) que podem ser ajustada(s) à situação estressante escolhida;

5a etapa - avaliação. Avaliar se o objetivo da etapa 2 foi atingido.

Se o objetivo estabelecido à partida não é atingido, é a ocasião de retomar o início do processo e fazer uma reflexão que permita ao cuidador tentar uma nova ação que será, por sua vez, também ela, avaliada. Esse processo sistemático permite ao cuidador orientar a sua reflexão até que o objetivo que fixou seja atingido.

O manual de aplicação tem as orientações para o enfermeiro sobre o conteúdo e os recursos para cada uma das cinco etapas do processo de gestão do estresse. O caderno do cuidador descreve o que o cuidador realiza em cada etapa, que corresponde a um encontro com o enfermeiro, bem como o que deve fazer até ao próximo encontro com esse profissional.

A operacionalização do programa inclui: cinco encontros semanais no domicílio entre o enfermeiro responsável pela prestação de cuidados ao idoso com o seu cuidador familiar com uma duração entre 30 a 45 minutos. Em cada encontro individual, o enfermeiro e o cuidador abordam cada uma das cinco etapas do processo de gestão do estresse. Ao longo do processo, é importante ter em conta a perceção que o cuidador tem da sua situação de prestação de cuidados. Esta perceção é a pedra angular da intervenção, com vista a favorecer a utilização de estratégias adaptativas ajustadas ao tipo da situação difícil/ estressante. No primeiro encontro, o enfermeiro oferece ao cuidador o "caderno para o cuidador" e explica a sua utilidade. No início de todos os encontros são relembradas as etapas do processo de gestão do estresse, o que foi concretizado na 
sessão anterior (exceto no primeiro encontro), o que é trabalhado no encontro presente e o que será feito na semana seguinte até ao próximo encontro.

Cada enfermeiro recebeu preparação prévia, em grupo ou individualmente, durante 2-3 sessões, com duração de 2 horas, sobre o protocolo de intervenção, e recebeu o material escrito (caderno do cuidador e manual de aplicação para si).

No fim da aplicação da intervenção, no mês 2, a investigadora conduziu uma entrevista semiestruturada junto aos enfermeiros e cuidadores familiares, para avaliar o programa sobre a ajuda da intervenção e a adequação dos materiais de apoio.

O protocolo de investigação foi aprovado pelo Diretor Executivo do respetivo Agrupamento e teve parecer favorável da Comissão de Ética para a Saúde da Administração Regional de Saúde de Lisboa e Vale do Tejo, 093/CES/ INV/2014. O consentimento informado foi assinado pelos participantes.

A análise dos dados foi qualitativa (do conteúdo das respostas de cuidadores e enfermeiros) e estatística. $\mathrm{Na}$ análise estatística, o tratamento e a análise dos dados foram obtidos por meio do software estatístico IBM-SPSS, versão 22. Análise descritiva das variáveis sociodemográficas e da história de cuidados foi obtida. Realizou-se a análise comparativa, para identificar associações entre variáveis de interesse por meio do teste $\mathrm{t}$ para amostras emparelhadas nas variáveis numéricas ou em alternativa o teste não paramétrico Wilcoxon. Na análise estatística, assumiu-se um nível de significância de 5\% ( $<<0,05)$.

A Grelha para avaliação do cuidador em risco foi aplicada como critério de elegibilidade do participante, conforme sugerido pelas autoras do citado programa psicoeducativo. Participante com escore igual ou superior a 16 , indicativo de elevado nível de estresse, foi excluído do estudo e aconselhado a procurar apoio especializado.

Questionário de caracterização sociodemográfica do cuidador/idoso e da história da prestação do cuidado, com instrumentos usados, avaliam os domínios do modelo de estresse $\operatorname{adotado}^{(12)}$.
Para avaliar a sobrecarga, aplicou-se a Burden Interview Scale, de 1983, adaptada para Portugal, que possui quatro categorias: impacto da prestação de cuidados, relação interpessoal, expectativas face ao cuidar e perceção de autoeficácia. A versão tem 22 itens. As possibilidades de resposta são: nunca (1), quase nunca (2), às vezes (3), muitas vezes (4) e quase sempre (5). À maior pontuação corresponde maior sobrecarga. A consistência interna é boa, com 0,96 (alfa de Cronbach) ${ }^{(16)}$.

Para avaliar o coping, aplicou-se o Carers' Assessment of Managing Index (CAMI) adaptado para Portugal. Esse questionário avalia a forma como cada cuidador familiar lida com as dificuldades percecionadas e quais os mecanismos de coping e a sua adequação e eficácia. Tem três domínios: lidar com problemas/resolução de problemas, perceção alternativa da situação e lidar com problemas de estresse. O cuidador indica se utiliza ou não a estratégia (1). Em caso afirmativo, deve referir se dá resultado (2), dá algum resultado (3) ou dá bastante resultado (4). À maior pontuação corresponde maior utilização/perceção da eficácia das estratégias de coping usadas pelo cuidador. A consistência interna é boa com 0,804 (alfa de Cronbach) ${ }^{(17)}$.

Para avaliar o bem-estar emocional do cuidador, o domínio mais avaliado são sintomas depressivos propostos pelo Centerfor Epidemiologic Studies of Depression Scale (CES-D), tendo sido usada a versão portuguesa, que propõe quatro fatores: afeto depressivo, afeto positivo, atividade somática e retardada e atividade interpessoal. Maior valor indica a intensidade dos sintomas da depressão. A consistência interna é boa com 0,85 (alfa de Cronbach) ${ }^{(12)}$.

\section{Resultados}

Dos 17 participantes, 13 completaram as 2 fases de avaliação antes e depois da intervenção (3 óbitos de idosos e 1 cuidador recusou-se a participar).

$\mathrm{Na}$ Grelha de avaliação do cuidador em risco, o valor da média $=$ mediana foi 11 e o Desvio Padrão (D.P.)=2,09, com máximo de 15 e mínimo 
6

Intervenção de enfermagem no estresse do cuidador familiar do idoso com dependência: estudo piloto

de 8 , em que nenhum dos cuidadores atingiu o nível de escore máximo (16) para ser excluído do estudo. Nos cuidadores, o sexo feminino foi o mais frequente; a média de idade foi 62,18 anos (DP=15,72); e o estado civil, casadas. Na situação de trabalho e escolaridade, foi mais frequente o cuidador aposentado e com o $3^{\circ}$ ciclo do ensino básico. Similarmente, estudo em Portugal descreve o perfil do cuidador familiar também como sendo do género feminino, com mais de 50 anos, casada, com baixa escolaridade, sem atividade profissional $^{(18)}$.

Nos idosos, a frequência é idêntica entre homens e mulheres, com idade média de 81,5 $(\mathrm{DP}=9,85)$. Na escolaridade, o mais frequente é $1^{\text {o }}$ ciclo do ensino básico. O estudo realizado ${ }^{(19)}$ com cuidadores familiares, os idosos cuidados tinham, em média, idade ligeiramente superior $(82,36$ anos; D.P.=7,84), igualmente poucos anos de escolaridade e são viúvos, ao contrário dos idosos cuidados deste estudo, que são maioritariamente casados.

$\mathrm{Na}$ história da prestação de cuidados, com maior frequência estão os cuidadores que cuidam em tempo, entre 1-3 anos, e em horas de cuidados diárias, mais de 10 h dedicadas ao cuidado. A prestação de cuidados é da responsabilidade de filhas e cônjuges; a maioria coabita com o idoso. Esses dados vão ao encontro de estudo sobre a sobrecarga de cuidadores familiares ${ }^{(20)}$.

A Tabela 1 apresenta as características sociodemográficas do cuidador e dados sobre a história do cuidar na avaliação baseline.

Tabela 1 - Distribuição dos cuidadores segundo características sociodemográficas e da história do cuidar na avaliação baseline. Lisboa, Portugal - 2015 ( $n=13)$

\begin{tabular}{|c|c|c|}
\hline $\begin{array}{l}\text { Características sociodemográfica do } \\
\text { cuidador }\end{array}$ & $\mathbf{n}$ & $\%$ \\
\hline \multicolumn{3}{|l|}{ Sexo do cuidador } \\
\hline Feminino & 8 & 61,5 \\
\hline Masculino & 5 & 38,5 \\
\hline Idade (anos), média (DP) & $62,18(15,72)$ & \\
\hline \multicolumn{3}{|l|}{ Estado civil do cuidador } \\
\hline Casado(a) & 7 & 53,8 \\
\hline Solteiro(a) & 4 & 30,8 \\
\hline Viúvo(a) & 1 & 7,7 \\
\hline Divorciado(a)/separado(a) & 1 & 7,7 \\
\hline \multicolumn{3}{|l|}{ Situação perante o trabalho } \\
\hline Empregado(a) & 3 & 23,1 \\
\hline Desempregado(a) & 1 & 7,7 \\
\hline Aposentado(a) & 8 & 61,5 \\
\hline Outra & 1 & 7,7 \\
\hline \multicolumn{3}{|l|}{ Escolaridade } \\
\hline $1^{\text {o }}$ ciclo do ensino básico & 3 & 23,1 \\
\hline 3o ciclo do ensino básico & 5 & 38,5 \\
\hline Ensino secundário & 3 & 23,1 \\
\hline Curso superior & 2 & 15,4 \\
\hline
\end{tabular}

Fonte: Elaboração própria.

A Tabela 2 apresenta as características sociodemográficas do idoso.

Tabela 2 - Características sociodemográficas do idoso. Lisboa, Portugal - 2015 (n=13) (continua)

\begin{tabular}{|c|c|c|}
\hline Caracterização sociodemográfica do idoso & $\mathbf{n}$ & $\%$ \\
\hline \multicolumn{3}{|l|}{ Sexo } \\
\hline Feminino & 7 & 53,8 \\
\hline Masculino & 5 & 46,2 \\
\hline
\end{tabular}


Tabela 2 - Características sociodemográficas do idoso. Lisboa, Portugal - 2015 (n=13)

(conclusão)

\begin{tabular}{|c|c|c|}
\hline Caracterização sociodemográfica do idoso & $\mathbf{n}$ & $\%$ \\
\hline Idade (anos), média (DP) & $81,47(c$ & \\
\hline \multicolumn{3}{|l|}{ Estado civil } \\
\hline Casado(a) & 7 & 53,8 \\
\hline Viúvo(a) & 4 & 30,8 \\
\hline Divorciado(a)/separado(a) & 2 & 15,4 \\
\hline \multicolumn{3}{|l|}{ Escolaridade } \\
\hline Sem escolaridade & 1 & 7,7 \\
\hline Sabe ler e escrever & 2 & 15,4 \\
\hline $1^{\text {o }}$ ciclo do ensino básico & 6 & 46,2 \\
\hline $2^{\circ}$ ciclo do ensino básico & 2 & 15,4 \\
\hline 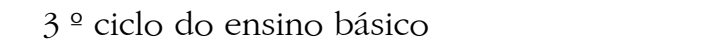 & 1 & 7,7 \\
\hline Ensino secundário & 1 & 7,7 \\
\hline \multicolumn{3}{|l|}{ História da prestação de cuidados } \\
\hline \multicolumn{3}{|l|}{ Grau de parentesco } \\
\hline Cônjugue & 4 & 30,8 \\
\hline Filho(a) & 6 & 46,2 \\
\hline Sobrinha & 2 & 15,3 \\
\hline Irmão & 1 & 7,7 \\
\hline \multicolumn{3}{|l|}{ Coabitação } \\
\hline $\operatorname{Sim}$ & 12 & 92,3 \\
\hline Não & 1 & 7,7 \\
\hline \multicolumn{3}{|l|}{ Há quanto tempo é cuidador } \\
\hline Menos de 6 meses & 2 & 15,4 \\
\hline Entre 6 meses e 1 ano & 1 & 7,7 \\
\hline Entre $1-3$ anos & 6 & 46,2 \\
\hline Entre 3-5 anos & - & - \\
\hline Entre 5-10 anos & 1 & 7,7 \\
\hline Há mais de 10 anos & 3 & 23,1 \\
\hline \multicolumn{3}{|c|}{ Número de horas diárias na prestação de cuidados } \\
\hline Entre $1-3$ horas & 1 & 7,7 \\
\hline Entre $3-5$ horas & 2 & 15,4 \\
\hline Entre 5-10 horas & 1 & 7,7 \\
\hline Mais de 10 horas & 9 & 69,2 \\
\hline
\end{tabular}

Fonte: Elaboração própria.

Nota: Sinal convencional utilizado:

- Dado numérico igual a zero não resultante de arredondamento.

Na avaliação das variáveis de resultado, entre o antes e o depois da intervenção, observou-se diferenças estatisticamente significativas na sobrecarga total $(\mathrm{p}=, 023)$ e nas categorias impacto da prestação de cuidados $(p=, 037)$ e expetativas face ao cuidar $(\mathrm{p}=, 023)$. Os cuidadores melhoraram as estratégias de coping, sobretudo na categoria perceção alternativa da situação. Observou-se também melhoria no bem-estar emocional; na categoria afeto positivo, a diferença foi estatisticamente significativa $(p=, 024)$. A Tabela 3 revela os dados obtidos antes (baseline) e depois da intervenção (T1). 
Tabela 3 - Distribuição dos cuidadores segundo a sobrecarga, o coping e sintomas depressivos avaliados na baseline e depois da intervenção (T1). Lisboa, Portugal - 2015 (n=13)

\begin{tabular}{|c|c|c|c|}
\hline Variável & $\begin{array}{c}\text { Baseline } \\
\text { Média (Desvio } \\
\text { Padrão) } \\
\end{array}$ & $\begin{array}{c}\text { T1 } \\
\text { Média (Desvio } \\
\text { Padrão) }\end{array}$ & $\begin{array}{l}\text { t emparelhado } \\
\text { Wilcoxon }\end{array}$ \\
\hline \multicolumn{4}{|l|}{ Sobrecarga } \\
\hline Impacto da prestação de cuidados & $30,69(11,24)$ & $24,84(6,84)$ & $\begin{array}{c}\text { temp }=2,340 \\
\mathrm{P}=, 037\end{array}$ \\
\hline Relação interpessoal & $9,15(3,48)$ & $7,76(3,24)$ & $\begin{array}{c}\text { temp }=1,737 \\
\mathrm{P}=, 108\end{array}$ \\
\hline Expectativas face ao cuidar & $16,53(3,12)$ & $14,07(3,88)$ & $\begin{array}{c}\text { temp }=2,606 \\
\mathrm{P}=, 023\end{array}$ \\
\hline Perceção de autoeficácia & $4,30(1,79)$ & $3,35(1,39)$ & $\begin{array}{c}\text { temp }=2,034 \\
\mathrm{P}=, 065\end{array}$ \\
\hline Total & $60,69(15,71)$ & $50,23(10,17)$ & $\begin{array}{c}\text { temp }=2,606 \\
\mathrm{P}=, 023\end{array}$ \\
\hline \multicolumn{4}{|l|}{ Coping } \\
\hline Resolução problemas & $41,07(7,44)$ & $42,23(7,35)$ & $\begin{array}{c}t e m p=, 233 \\
\mathrm{P}=, 820\end{array}$ \\
\hline Perceção alternativa situação & $40,92(7,064)$ & $43,38(10,36)$ & $\begin{array}{c}\text { temp=-,760 } \\
\mathrm{P}=, 462\end{array}$ \\
\hline Lidar com sintomas de estresse & $20,07(6,04)$ & $20,84(6,01)$ & $\begin{array}{c}\text { temp }=-1,949 \\
\mathrm{P}=, 075\end{array}$ \\
\hline Total & $99,62(15,11)$ & $105,69(19,12)$ & $\begin{array}{c}\text { temp }=-1,774 \\
\mathrm{P}=, 101\end{array}$ \\
\hline \multicolumn{4}{|l|}{ Bem-estar emocional } \\
\hline Afeto deprimido & $8,00(5,73)$ & $6,0(4,43)$ & $\begin{array}{c}\text { temp }=1,747 \\
\mathrm{P}=, 111\end{array}$ \\
\hline Afeto positivo & $8,38(3,28)$ & $6,50(2,97)$ & $\begin{array}{c}\text { temp }=2,327 \\
\mathrm{P}=, 040\end{array}$ \\
\hline Atividade somática retardada & $4,00(3,64)$ & $4,55(3,8)$ & $\begin{array}{c}\text { temp=-,193 } \\
\mathrm{p}=, 851\end{array}$ \\
\hline Atividade Interpessoal & $2,25(1,89)$ & $1,50(0,71)$ & $\begin{array}{c}\text { Wilcoxon } \\
\mathrm{p}=, 102\end{array}$ \\
\hline Score total & $20,77(12,14)$ & $16,42(9,41)$ & $\begin{array}{l}\text { Wilcoxon } \\
\mathrm{P}=, 66\end{array}$ \\
\hline
\end{tabular}

Fonte: Elaboração própria.

$\mathrm{Na}$ análise qualitativa dos dados sobre a opinião dos cuidadores familiares acerca da ajuda da intervenção, as respostas foram claramente positivas. Dos 13 participantes, apenas 1 manifestou opinião negativa e outro participante respondeu com opinião ambivalente. Seguem alguns exemplos da citação (verbatim) da análise qualitativa.

Opinião positiva:

A intervenção ajuda a encarar melbor a situação de cuidador e a encontrar novos caminhos de como continuar. (F; 62 anos; filha).

A situação de cuidador mantêm-se, mas a forma como a vejo é mais calma e serena. (M; 40 anos; sobrinha).
A intervenção ajudou a estar preparada para outras dificuldades que vão surgir e que sei que me esperam. (N; 50 anos; filha).

Aprendi a deitar e a levantar para a cadeira com menos esforço para as costas. Já não preciso de estar à espera de ajuda. (J; 75 anos; cônjuge).

A intervenção ajudou a perceber que, se mudar a minha forma de agir, o que está à volta melhora. (A; 60 anos; filha).

Opinião negativa:

A intervenção não ajudou em nada. Fez-me pensar em assuntos que estavam resolvidos e agora vieram à memória. (L; 78 anos; irmão). 
Opinião ambivalente:

A intervenção ajudou e não ajudou. Parar para pensar não me ajuda [...] lembra-me que estou sozinbo nisto e como será no futuro, o que será dela [Esposa]? Os filbos não querem saber [...] mas foi bom ter alguém para falar, senti-me apoiado. (A; 78 anos; cônjuge).

Sobre o material de apoio de suporte ao programa, cinco cuidadores consideraram o conteúdo do caderno do cuidador adequado e oito referiram o conteúdo extenso, sobretudo na $3^{\underline{a}}$ etapa.

Dos oito enfermeiros, quatro opinaram sobre a avaliação e implementação da intervenção, obtendo-se opinião com respostas que oscilaram entre aspetos positivos e negativos. Vejam-se exemplos de citações de aspetos positivos:

Enfermeiro conbece melhor o cuidador. Há uma maior proximidade com o cuidador, maior compreensão das dificuldades que antes não tínhamos e criamos empatia. (E1).

O momento que estamos com o cuidador é formal. O cuidador sente-se cuidado, tem a atenção do enfermeiro [...] aceita melhor o que lhe é dito. (E2).

Com a intervenção, damos ferramentas para o cuidador encontrar os recursos para resolução de problemas, dando-lhe empowerment. (E3).

Se queremos o cuidar bolístico não é só o doente em si; o outro que cuida também deve ser cuidado, para o bem do doente. (E4).

Exemplos de citações negativas:

Estamos pressionados pelo tempo dedicado a cada visita domiciliária. (E1; E2; E3; E4).

Se o tempo prolongar além do previsto para cada uma das visitas, isso tem implicações na gestão dos cuidados aos outros doentes por quem sou responsável e aos cuidados prestados aos outros doentes pelos colegas que esperam por nós, devido ao transporte partilhado. (E3).

Há coisas muito enraizadas no cuidador que já não mudam, ou levam mais tempo. (E4).

Muitos idosos ficam desconfiados, com receio de falar de alguns aspetos da vida familiar e comprometerem a família por ausência de apoio. (E1).

Por vezes, os cuidadores mais velhos perdem a noção do que é para fazer; é necessário lembrar o que foi já feito e por vezes voltar atrás. (E2; E4).

Relativamente ao material de apoio, a opinião geral sobre o caderno do cuidador recai no excesso de informação. Consequentemente, os cuidadores mais velhos e com menos anos de escolaridade perdem-se com o número de páginas.
Sobre o manual para aplicação, tem o conteúdo adequado para a implementação do programa pelos enfermeiros, mas o seu transporte diário, nas visitas domiciliárias, é pouco prático.

\section{Discussão}

Os resultados deste estudo revelam que os cuidadores percebem menos sobrecarga associada ao caregiving, usam mais estratégias de coping e indicam mais bem-estar após a intervenção com base num programa psicoeducativo de gestão do estresse. Tais resultados validam a hipótese do estudo, encontrando-se, para a sobrecarga, diferenças estatisticamente significativas. Tal dado parece confirmar que a intensidade de horas dedicadas à prestação de cuidados leva à sobrecarga intensa ${ }^{(21)}$ que diminuiu para ligeira após a intervenção. Este dado valida a importância de as intervenções deverem ser pró-activas em vez de reativas para promoção da saúde dos cuidadores familiares ${ }^{(22)}$.

Os resultados no aumento das estratégias do coping são similares ao estudo publicado pelas autoras do programa ${ }^{(23)}$. Tratando-se de um programa de gestão do estresse, após a sua aplicação na forma de intervenção, as estratégias de coping aumentaram nos 3 domínios avaliados pelo mesmo instrumento no estudo realizado pelas autoras, com maior aumento no domínio da perceção alternativa da situação. Tal resultado parece significar que o cuidador, na $3^{\text {a }}$ etapa do processo de gestão do estresse, refletiu nas estratégias habitualmente utilizadas para a situação identificada por ele como difícil na prestação de cuidados e percebeu outras estratégias de coping mais adaptadas ao caso, parecendo revelar a capacidade de aprender e pôr em prática novas estratégias de coping ${ }^{(13)}$.

O resultado sobre o bem-estar do cuidador, na forma de sintomas depressivos, não foi avaliado em estudos publicados pelas autoras do programa. No entanto, esta é uma variável frequentemente usada nos estudos internacionais, na avaliação do efeito das intervenções sobre o bem-estar emocional do cuidador. A sua utilização neste estudo revelou tratar-se de uma 
variável sensível à intervenção de enfermagem. Assim, o resultado após a intervenção foi melhoria do bem-estar, à semelhança de estudo piloto realizado na Austrália ${ }^{(24)}$. Acrescente-se que, na categoria afeto positivo, a diferença foi estatisticamente significativa, revelando que o cuidador melhorou a valorização pessoal e o sentimento de felicidade.

Apesar do número limitado de observações, os dados qualitativos deste estudo revelaram resultados que merecem reflexão e podem ajudar em estudos seguintes. A verbalização dos cuidadores sobre a ajuda da intervenção aplicada pelos enfermeiros encontra fundamento na opinião de autor $^{(25)}$, ao referir que os valores humanistas e os conhecimentos psicossociais que a enfermeira tem sobre a pessoa são fundamentais para compreender e conhecer o seu comportamento adaptativo ou inadequado e ajudá-la a utilizar a totalidade do seu potencial para encontrar a melhor qualidade de vida possível após intervenções promotoras de adaptação ao estresse.

A dificuldade apontada à implementação da intervenção pelos enfermeiros recai, sobretudo, sobre a falta de tempo para aplicação na prática diária, uma vez que a intervenção contemplava um encontro de pelo menos 30 minutos semanais, durante 5 semanas. Este dado é consistente com os dados de estudo(26) que salienta esse fator como resistência à aplicação duradoura de uma intervenção de enfermagem junto dos cuidadores.

O número crescente de situações de incapacidade decorrentes do envelhecimento da população suscita reflexão sobre os cuidadores familiares como elementos mediadores, os quais devem ser entendidos como recurso institucional e, dessa forma, importante para a continuidade dos cuidados. Para tal, o enfermeiro, ao assumir a maioria dos cuidados domiciliários ${ }^{(27)}$, ajuda o cuidador familiar a prosseguir o seu papel social após uma intervenção, com base num programa, centrada no cuidador e nas suas dificuldades.
É fundamental, no que se refere à estrutura dos cuidados de enfermagem no domicílio, que, no tempo dedicado à visita do enfermeiro, seja formalmente contemplado o tempo para intervir junto do cuidador familiar, pois a investigação evidencia os benefícios para a díade cuidador/ idoso cuidado.

\section{Conclusão}

Este estudo piloto avaliou a eficácia da intervenção de enfermagem estruturada com base num programa psicoeducativo de gestão do estresse associado ao caregiving. Mesmo com um número reduzido de participantes, os resultados estatísticos apontam para a melhoria da sobrecarga, do coping e do bem-estar do cuidador familiar após a intervenção, 2 meses após o início dos trabalhos.

A análise qualitativa revelou a necessidade de sintetizar a informação no caderno do cuidador e explorar com os enfermeiros novas estratégias de aplicação da intervenção. Também forneceu informação útil sobre a aceitação da intervenção. Assim, pôde-se concluir que os cuidadores familiares revelaram que a intervenção ajudou-os na aprendizagem de novas estratégias de coping, perspectivando a continuidade do seu papel. Por parte dos enfermeiros, a intervenção ajudou-os a conhecer as dificuldades do cuidador, facilitando a abordagem holística do cuidado centrado no cuidador e no idoso.

No futuro sugere-se um estudo com a inclusão de maior número de participantes e de grupo de controlo, para comparar os resultados entre o grupo experimental e o grupo controlo.

\section{Colaborações:}

1. concepção, projeto, análise e interpretação dos dados: Laura Maria Monteiro Viegas;

2. redação do artigo, revisão crítica relevante do conteúdo intelectual: Laura Maria Monteiro Viegas, Ana Alexandre Fernandes e Maria dos Anjos Pereira Lopes F. Veiga; 
3. aprovação final da versão a ser publicada: Ana Alexandre Fernandes e Maria dos Anjos Pereira Lopes F. Veiga.

\section{Referências}

1. Fernandes A. Saúde e doença e (r)evolução demográfica. In: Fonseca A, editors. Envelhecimento, saúde, doença: novos desafios para a prestação de cuidados a idosos. Lisboa: Coisas de Ler; 2014. p. 7-26.

2. Portugal. Instituto Nacional de Estatística. Censos. Resultados definitivos. Região Norte Portugal - 2011 [Internet]. Lisboa; 2012 [cited 2017 Nov 19]. Available from: http://censos.ine. $\mathrm{pt} / \mathrm{xportal} / \mathrm{xmain}$ ? xpid=CENSOS\&xpgid $=$ ine $_{-}$ censos_publicacao_det\&contexto= pu\&PUBLICACOESpub_boui=156638623\& P UBLICACOES modo $=2 \& \operatorname{selTab}=\mathrm{tab} 1 \&$ pcensos $=61969554$

3. Portugal. PORDATA. Esperança de vida à nascença total e por sexo [Internet]. Lisboa; Fundação Manuel do Santos [cited 2018 Apr 6]. Available from: https://www.pordata.pt/Europa/Esperan $\%$ c3\%a $7 a+d e+v i d a+\% c 3 \% a 0+$ nascen $\% c 3 \%$ a7a+total+e+por+sexo-1260-209738

4. Family Caregiver Alliance, editors. Caregiver Assessment: Voices and Views from the Field. Report from a National Consensus Development Conference. San Francisco: National Center on Caregiving at Family Caregiver Alliance; 2006.

5. Lage I, Araújo O. A construção discursiva do cuidado informal a idosos dependentes: perceções, constrangimentos e práticas. In: Fonseca A, editors. Envelhecimento, saúde, doença: novos desafios para a prestação de cuidados a idosos. Lisboa: Coisas de Ler; 2014. p. 219-62.

6. Morris TL, Edwards L. Family Caregivers. In: Lubkin I, Larsen P, editors. Chronic illness. Impact and interventions. 6nd ed. Sudbury (CAN): Jones and Bartlett Publishers; 2006. p. 253-84.

7. Williams L. Theory of caregiving dynamics. In: Smith M, Liehr P, editors. Middle range Theory for nursing. New York: Springer Publishing Company; 2008. p. 261-75.

8. Au A, Gallagher-Thompson D, Wong MK, Leung J, Chan WC, Chan CC, et al. Behavioral activation for dementia caregivers: scheduling pleasant events and enhancing communications. Clin Interv Aging. 2015 Mar;10:611-9.
9. Couto A, Hell C, Lemos I, Castro E. Cuidado domiciliar sob ótica de idosos dependentes: contribuições para a enfermagem. Rev baiana enferm. 2016 [cited 2017 Nov 29];30(4):1-12. Available from: DOI 10.18471/rbe.v30i4.16068

10. Steffen A, Gant JR, Gallagher-Thompson D. Reducing psychosocial distress in family caregivers. In: Gallaguer-Thompson D, Steffen M, Thompson L, editors. Handbook of behavioral and cognitive therapies with older adults. New York: Spinger Publishing Company; 2008. p.102-17.

11. Suhonen R, Välimäki M, Leino-Kilpi H. A review of outcomes of individualized nursing interventions on adult paciente. J Clin Nurs. 2008 Apr;17(7):843-60.

12. Zarit S. Assessment of family Caregivers: a research perspective. In: Family Caregiver Alliance, editors. Caregiver assessment: principles, guidelines and strategies for change. Report from a National Concensus Development Conference. San Francisco: National Center on Caregiving at Family Caregiver Alliance; 2006 (Vol. 2). p.12-37.

13. Lazarus RS, Folkman S. Stress, appraisal and coping. New York: Springer; 1984.

14. De la Cuesta C. Strategies for the relief of burden in advanced dementia care-giving. J Adv Nurs. 2011 [cited 2017 Nov 20];67:1790-99. Available from: Doi.10.1111/j.1365-2648.2010.05607.x

15. Ducharme F, Trudeau D, Ward J. Manuel de l'intervenant - gestion du stress - Programme psychoéducatif de gestion du stress destine aux proches-aidants d'un parent âgé à domiicile. Montréal: Institut Universitaire de Gériatrie de Montréal, Université de Montréal; 2008.

16. Sequeira C. Difficulties, coping strategies, satisfaction and burden in informal Portuguese caregivers. J Clin Nurs. 2013 Feb [cited 2017 Nov 25];22:491-500. Available from: Doi: 10.1111/ jocn.12108

17. Brito ML. A saúde mental dos prestadores de cuidados a familiares idosos [dissertação]. Porto: Faculdade de Medicina, Universidade do Porto; 2000.

18. Carrilho da Cunha M. Impacto positivo do acto de cuidar no cuidador informal do idoso: um estudo exploratório nos domicílios do Concelho de Gouveia [dissertação]. Lisboa: Faculdade de Psicologia, Universidade de Lisboa; 2011.

19. Costa A. A família cuidadora perante a dependência do seu familiar idoso [dissertação]. Lisboa: 
Faculdade de Ciências Médicas, Universidade Nova de Lisboa; 2012.

20. Pereira S. Cuidar de idosos dependentes: a sobrecarga dos cuidadores familiares [dissertação]. Braga: Universidade Católica Portuguesa; 2015.

21. Ory M, Yee J, Tennstedt S, Schulz R. The extent and Impact of dementia care: unique Challenges Experienced by Family Caregivers. In: Shultz R, editors. Handbook on dementia Caregiving: Evidence Base Interventions for Family Caregivers. New York: Springer Publishing Company; 2000. p. $1-32$.

22. Ducharme F, Dubé V, Lévesque L, Saulnier D, Giroux F. An online stress Management Training Program as a Supportive Nursing Intervention for Family Caregiver of an Elderly Person. Canadian J Nurs Inform [serial on the internet]. 2011 [cited 2016 Aug 20];6(2). Available from: http://cjni.net/ journal/?p=1344

23. Ducharme F, Lebel P, Lachance L, Trudeau D. Implementation and effects of an individual stress management intervention for family caregivers of an elderly relative living home: a mixed research design. Res Nurs Health. 2006;29:427-41.
24. Leone D, Carragher N. Santalucia Y, Draper B, Thompson LW, Shanley C, et al. A pilot of an intervention delivered to Chinese-and Spanishspeaking carers of people with dementia in Australia. Am J Alzheimers Dis Other Demen. 2014 Feb;29(1):32-7.

25. Watson J. Nursing. The Philosophy and Science of Caring. Boulder (CO): University Press of Colorado; 1985.

26. Ferré-Grau C, Sevilla-Casado M, Lleixá-Fortuño M, Aparicio-Casals MR, Cid-Buera D, Rodero-Sanchez $\mathrm{V}$, et al. Effectiveness of problem-solving technique in caring for Family caregivers: a clinical trial study in an urban area of Catalonia (Spain). J Clin Nurs. 2014 Jan;23(1-2):288-95.

27. Portugal. Observatório Português dos Sistemas de Saúde. Relatório de Primavera 2015. Acesso aos cuidados de saúde. Um direito em risco? [Internet]. Lisboa; 2015 [cited 2017 July 10]. Available from: http://www.opss.pt/sites/opss.pt/files/Relatorio Primavera2015.pdf

Recebido: 28 de dezembro de 2017 Aprovado: 7 de fevereiro de 2018

Publicado: 23 de maio de 2018

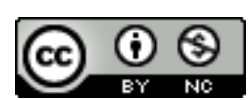

A Revista Baiana de Enfermagem utiliza a Licença Creative Commons - Atribuição-NãoComercial 4.0 Internacional. https://creativecommons.org/licenses/by-nc/4.0/ Este artigo é de acesso aberto distribuído sob os termos da Licença Creative Commons (CC BY-NC). Esta licença permite que outros remixem, adaptem e criem a partir do seu trabalho para fins não comerciais e, embora, os novos trabalhos tenham de lhe atribuir o devido crédito e não possam ser usados para fins comerciais, os usuários não têm de licenciar esses trabalhos derivados sob os mesmos termos. 